\title{
MafA is critical for maintenance of the mature beta cell phenotype in mice
}

\author{
Wataru Nishimura $\cdot$ Satoru Takahashi $\cdot$ Kazuki Yasuda
}

Received: 31 August 2014 / Accepted: 13 November 2014 / Published online: 13 December 2014

(C) Springer-Verlag Berlin Heidelberg 2014

\begin{abstract}
Aims/hypothesis The plasticity of adult somatic cells allows for their dedifferentiation or conversion to different cell types, although the relevance of this to disease remains elusive. Perturbation of beta cell identity leading to dedifferentiation may be implicated in the compromised functions of beta cells in diabetes, which is a current topic of islet research. This study aims to investigate whether or not v-Maf musculoaponeurotic fibrosarcoma oncogene family, protein A (MafA), a mature beta cell marker, is involved in maintaining mature beta cell phenotypes.

Methods The fate and gene expression of beta cells were analysed in Mafa knockout (KO) mice and mouse models of diabetes in which the expression of MafA was reduced in the majority of beta cells.

Results Loss of MafA reduced the beta to alpha cell ratio in pancreatic islets without elevating blood glucose to diabetic levels. Lineage tracing analyses showed reduced/lost expression of insulin in most beta cells, with a minority of the former beta cells converted to glucagon-expressing cells in Mafa KO
\end{abstract}

Electronic supplementary material The online version of this article (doi:10.1007/s00125-014-3464-9) contains peer-reviewed but unedited supplementary material, which is available to authorised users.

W. Nishimura $(\square) \cdot K$. Yasuda $(\varangle)$

Department of Metabolic Disorders, Diabetes Research Center,

Research Institute, National Center for Global Health and Medicine,

1-21-1 Toyama, Shinjuku-ku, Tokyo 162-8655, Japan

e-mail: wnishimura@jichi.ac.jp

e-mail: kyasuda@ri.ncgm.go.jp

W. Nishimura

Division of Anatomy, Bio-imaging and Neuro-cell Science,

Jichi Medical University, Shimotsuke, Tochigi, Japan

S. Takahashi

Department of Anatomy and Embryology, University of Tsukuba,

Tsukuba, Ibaraki, Japan mice. The upregulation of genes that are normally repressed in mature beta cells or transcription factors that are transiently expressed in endocrine progenitors was identified in Mafa KO islets as a hallmark of dedifferentiation. The compromised beta cells in $d b / d b$ and multiple low-dose streptozotocin mice underwent similar dedifferentiation with expression of Mafb, which is expressed in immature beta cells.

Conclusions/interpretation The maturation factor MafA is critical for the homeostasis of mature beta cells and regulates cell plasticity. The loss of MafA in beta cells leads to a deeper loss of cell identity, which is implicated in diabetes pathology.

Keywords Beta cells $\cdot$ Cell plasticity $\cdot$ Dedifferentiation · Lineage tracing $\cdot$ MafA

$\begin{array}{ll}\text { Abbreviations } \\ \text { BrdU } & \text { 5'-Bromo-2'-deoxyuridine } \\ \text { CHGA } & \text { Chromogranin A } \\ \text { DNMT } & \text { DNA methyltransferase } \\ \text { GFP } & \text { Green fluorescent protein } \\ \text { HFD } & \text { High fat diet } \\ \text { KO } & \text { Knockout } \\ \text { MafA } & \text { v-Maf musculoaponeurotic fibrosarcoma } \\ & \text { oncogene family, protein A } \\ \text { MafB } & \text { v-Maf musculoaponeurotic fibrosarcoma } \\ & \text { oncogene family, protein B } \\ \text { MLD } & \text { Multiple low-dose } \\ \text { NeuroD1 } & \text { Neurogenic differentiation 1 } \\ \text { PDX1 } & \text { Pancreatic and duodenal homeobox 1 } \\ \text { SYP } & \text { Synaptophysin } \\ \text { RIP } & \text { Rat insulin 2 promoter } \\ \text { STZ } & \text { Streptozotocin } \\ \text { TEM } & \text { Transmission electron microscopy } \\ \text { YFP } & \text { Yellow fluorescent protein }\end{array}$




\section{Introduction}

Increasing evidence has shown that cell differentiation is a reversible and dynamic state in various organs in vivo [1, 2]. Cell plasticity has been intensively investigated in adult pancreatic endocrine cells. Lineage tracing analyses of adult pancreatic cells for various in vivo conditions clearly demonstrate that conversion between different pancreatic cell types occurs in adults through changes in the expression of transcription factors that are critical for endocrine cell differentiation [3-8]. These findings are supported by studies reporting that the forced expression of transcription factors convert cell types [9-11]. In addition, recent studies have identified factors critical for the maintenance of a mature beta cell phenotype. The loss of a mature beta cell phenotype results in the dedifferentiation of beta cells [8, 12-16], which may be implicated in compromised beta cell function in diabetes, although the molecular mechanisms remain unknown.

Differentiation studies have suggested that maturity status is involved in the dedifferentiation process in committed airway secretory cells [17], as well as in terminally differentiated B lymphocytes [18] and the nuclear reprogramming process [19]. These results suggest that differentiation is actively maintained by maturation factors in mammalian cells. The insulin gene transcription factor v-Maf musculoaponeurotic fibrosarcoma oncogene family, protein A (MafA) is expressed in mature beta cells [20-22] and is not involved in endocrine specification during embryonic development [23]. The ectopic expression of MafA (Mafa), pancreatic and duodenal homeobox 1 (PDX1 $[P d x 1]$ ) and neurogenic differentiation 1 (NeuroD1 [Neurod1]) or neurogenin 3 (Ngn3, also known as Neurog3) converts adult pancreatic acinar cells or liver cells into insulin-expressing cells $[11,24]$. In addition, reduced MafA expression is a harbinger of beta cell dysfunction in human type 2 diabetes $[25,26]$. In the present study, we examined the role of MafA in the plasticity and homeostasis of beta cells in mice.

\section{Methods}

Animals The Animal Care and Use Committee of the National Center for Global Health and Medicine approved all the animal experiments. The Mafa knockout (KO) [27] and $M a f b$-green fluorescent protein (Mafb-GFP) [28] mice analysed in this study were maintained in ICR and C57BL/ 6J backgrounds (Japan SLC, Hamamatsu, Shizuoka, Japan), respectively. The Mafa $\mathrm{KO}$ mice were also maintained in a C57BL/6J background after backcrossing for more than eight generations, and these mice were mated with rat insulin 2 promoter (RIP) CreER mice (Jackson Laboratory, Bar Harbor, ME, USA, stock number: 008122) and Rosa-yellow fluorescent protein (YFP) mice (Jackson Laboratory, stock number: 006148) for the lineage tracing study. The $d b / d b$ and control $\mathrm{db} / \mathrm{m}$ mice were obtained from CLEA Japan (Tokyo, Japan). The genotyping of the mice described above with the primers listed in electronic supplementary material (ESM) Table 1 was performed using $\mathrm{NaOH}$ extraction methods [29].

Regarding the lineage tracing studies, RIP-CreER;RosaYFP mice were injected intraperitoneally with tamoxifen (Sigma-Aldrich, St Louis, MO, USA) at $5 \mathrm{mg} / 20 \mathrm{~g}$ body weight for four consecutive days. For the beta cell lineage tracing analysis in mouse models of diabetes, a low dose (50 mg/kg body weight) of streptozotocin (STZ; SigmaAldrich) was injected intraperitoneally for five consecutive days 1 week after the injection of tamoxifen. The mice fed a high-fat diet (HFD) were generated with HFD 32 (CLEA Japan).

For glucose tolerance testing, $2 \mathrm{~g} / \mathrm{kg}$ body weight of glucose was injected intraperitoneally after the deprivation of food for 14-16 h. With the exception of those experiments using multiple low-dose (MLD) STZ-induced diabetic RIPCreER;Rosa-YFP mice and their controls, male mice or islets from male mice were analysed in this study.

Immunohistochemistry Immunostaining analyses were performed on paraffin-embedded sections as previously described [29] with the primary antibodies listed in ESM Table 2. The analyses of immunohistochemistry that included lineage tracing were based on confocal imaging. Regarding the quantification, sections were analysed at $200 \mu \mathrm{m}$ intervals using the National Institutes of Health ImageJ 1.46r (http:// imagej.nih.gov/ij/).

Transmission electron microscopy Isolated islets on culture dishes were fixed for transmission electron microscopy (TEM) observations according to Shirato et al [30] with slight modifications.

Quantitative RT-PCR of isolated islets Mouse islets were isolated using collagenase digestion as previously described [29]. Quantitative PCR amplification of cDNA from mouse islets was performed using the TaqMan universal PCR master mix core reagent kit with the probes listed in ESM Table 3, and the analysis was performed using an ABI Prism 7900 and Step One Plus (Applied Biosystems, Foster City, CA, USA); the $C_{t}$ values were measured in duplicate. The mRNA was quantified with normalisation to beta actin expression using the $2^{-\Delta \Delta C_{t}}$ method. The data are presented as mean \pm SEM, and statistical significance was determined using a two-tailed unpaired Student's $t$ test.

5'-Bromo-2'-deoxyuridine incorporation study A dose of $100 \mathrm{mg}$ 5'-bromo-2'-deoxyuridine (BrdU)/kg body weight (Sigma-Aldrich) was injected intraperitoneally into the mice, 
which was followed by ad libitum feeding for $24 \mathrm{~h}$. BrdU incorporation in the pancreas was analysed by immunofluorescence.

\section{Results}

Mafa loss primarily results in a reduced beta cell/alpha cell ratio To examine the effects of reduced MafA levels on beta cells, pancreatic sections from Mafa KO mice and their littermates were analysed by immunohistochemistry over time. Mafa KO mice had normal islet morphology at birth (Fig. 1a, b); however, increased intra-islet alpha cells were observed as early as 4 weeks of age (Fig. 1c, d). Insulin immunoreactivity in the beta cells was reduced in combination with a relative increase in the number of alpha cells from approximately 8 weeks of age (Fig. 1e, f). Cells with various levels of insulin expression and an increased population of cells that were not labelled with any pancreatic hormones were observed in Mafa KO islets at 10-12 weeks of age (Fig. 1g-1) [27]. Despite these changes in islet morphology, fasting blood glucose in adult Mafa $\mathrm{KO}$ mice did not reach diabetic levels, even at 48 weeks of age; however, these mice were glucose-intolerant (Fig. 1m-q, ESM Fig. 1a-h) [22]. Impaired dithizone staining and reduced insulin content were observed in the islets of Mafa KO mice (Fig. 1r-u). A TUNEL assay showed few apoptotic beta cells both in Mafa KO mice and in wild-type pancreatic sections (Fig. 1v).

Most cells in the Mafa KO islets at 12 weeks of age were stained with synaptophysin (SYP) or chromogranin A (CHGA), a committed endocrine cell marker, but showed reduced or no insulin expression (Fig. 2a-d). Moreover, TEM of the Mafa KO islets detected numerous vesicles without insulin granules in beta cells (Fig. 2e, f). These results suggest that in the Mafa $\mathrm{KO}$ mice beta cells vesicles formed but were empty because of reduced insulin expression. These insulin-negative 'empty' endocrine cells and the reduced beta to alpha cell ratios observed in the Mafa KO islets were rarely found in HFD mice from 4-12 weeks of age, although the HFD mice had blood glucose levels similar to or higher than those of the Mafa KO mice. In addition, the HFD islets exhibited no reduction in Mafa mRNA expression (ESM Fig. 2). These results suggest that the loss of MafA rather than elevated blood glucose levels, was primarily responsible for abnormal islet morphology in the adult Mafa KO mice, which is also a characteristic change in type 2 diabetes. The results also point to the importance of MafA in maintaining a mature beta cell phenotype.

MafA is an intrinsic factor for beta cell homeostasis To examine whether or not the $\mathrm{SYP}^{+}$insulin ${ }^{-}$or $\mathrm{CHGA}^{+}$insulin ${ }^{-}$ cells originated from beta cells, a lineage tracing study of insulin-expressing cells in MafA $\mathrm{KO}$ mice was performed using the MafaKO;RIPCreER;Rosa-YFP mice. In these mice, tamoxifen injection induces Cre recombinase specifically in insulin-expressing cells and causes YFP expression at the Rosa locus. YFP enables the tracking of beta cell fates (Fig. 2 g). More than $90 \%$ of the beta cells were labelled with YFP in the system used in this study (ESM Fig. 3a-d). Tamoxifen was injected into MafaKO;RIPCreER;Rosa-YFP mice and control wild-type;RIPCreER;Rosa-YFP mice at 4 weeks of age, and these mice were analysed at 12 weeks of age. Confocal imaging analysis of immunohistochemistry showed beta cells clearly labelled with YFP in the MafaKO;RIPCreER;Rosa-YFP mice (Fig. 2h-k), suggesting that insulin promoter activity and insulin expression (Fig. 1c, d) in the beta cells were not affected by MafA loss up to 4 weeks of age. Collectively, these results suggest that MafA is dispensable for beta cell formation in embryonic and neonatal pancreas tissues, although it remains unclear whether or not the beta cells in the pancreas of 4 week old Mafa KO mice were mature.

At 12 weeks of age, impaired insulin expression was observed in many YFP-expressing cells in the Mafa KO pancreas tissues (Fig. 2h, i), although the fasting blood glucose levels of the MafaKO;RIPCreER;Rosa-YFP mice did not reach diabetic levels (Fig. 21-n). Interestingly, in the MafaKO;RIPCreER;Rosa-YFP pancreas a small proportion of $\mathrm{YFP}^{+}$cells derived from the beta cells expressed glucagon (Fig. 2j,k). Immunohistochemistry quantification revealed that $2.6 \%$ of the total glucagon ${ }^{+}$cells expressed YFP $(n=$ 991), whereas these glucagon ${ }^{+} \mathrm{YFP}^{+}$cells were not observed in controls $(n=361)$. These results suggest that most beta cells at 4 weeks of age had lost insulin expression, and only a few of these cells were converted to glucagon ${ }^{+}$cells between 4 and 12 weeks of age with MafA loss. Although the proportion of glucagon $^{+}$cells in the islets remained high (ESM Fig. 3e), glucagon $^{+}$cell proliferation was not accelerated in the Mafa $\mathrm{KO}$ islets compared with the control islets (ESM Fig. $3 \mathrm{f}-\mathrm{h}$ ), which suggests that reduced beta cell proliferation [31, 32], in addition to cell conversion, may contribute to the increased proportion of glucagon ${ }^{+}$cells in Mafa $\mathrm{KO}$ islets.

Transcriptional changes in the islets of Mafa KO mice An mRNA expression analysis of the Mafa $\mathrm{KO}$ islets identified selective reduction in the expression of molecules critical for beta cell functioning, such as Ins1, Ins2, Glut2 (also known as Slc2a2), ZnT8 (also known as Slc30a8), Pcsk1, Vdr, Sytl4, Ucn3 and Maob, although the expression of Gck, Kir6.2 (also known as Kcnj11) and Surl (also known as $A b c c 8$ ), which are critical for glucose-stimulated insulin secretion, did not decrease (Fig. 3a). Expression of the transcription factors important for the maintenance of beta cells differentiation, such as PDX1, NeuroD1, paired box protein 6 (PAX6), forkhead box protein $\mathrm{O} 1$ (FOXO1) and Nkx6.1 $[8,12,33]$, was also mildly 


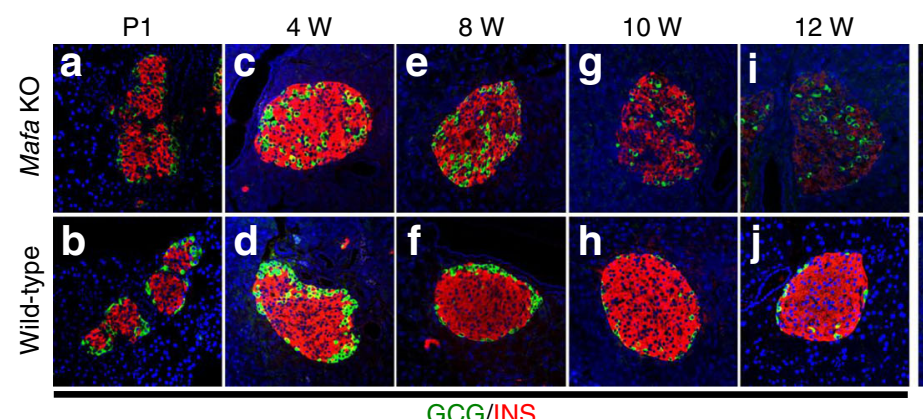

GCG/INS
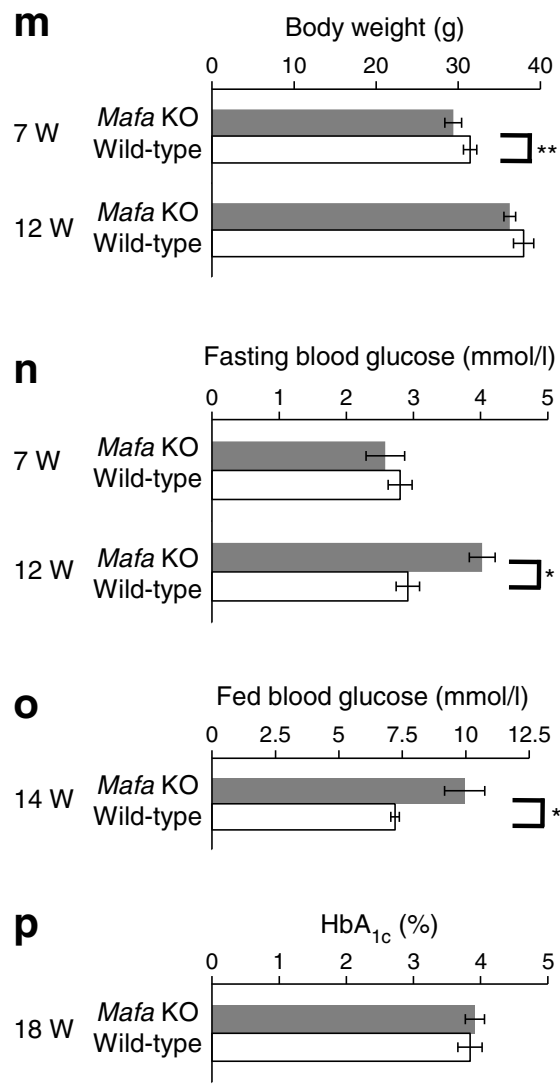

Fig. 1 Abnormal islet morphology in the Mafa KO mice. (a-I) Representative islet of the Mafa KO and wild-type mice stained for insulin (INS; red) and glucagon (GCG; green) at postnatal day $1(\mathbf{a}: n=4 ; \mathbf{b}: n=3)$ and at $4(\mathbf{c}: n=3 ; \mathbf{d}: n=5), 8(\mathbf{e}: n=5 ; \mathbf{f}: n=5), 10(\mathbf{g}: n=5 ; \mathbf{h}: n=5)$ and 12 (i: $n=9 ; \mathbf{j}: n=12)$ weeks (W) of age. (k, I) Islets of Mafa $\mathrm{KO}(n=9)$ and wild-type $(n=12)$ mice stained with a cocktail of non-beta cell hormones, glucagon, somatostatin (SOM) and pancreatic polypeptide (PP) (green) and insulin (red) at 12 weeks of age. Arrows indicate cells that were not labelled with any pancreatic hormones in Mafa KO islets. (m) Body weight, (n) fasting blood glucose and (o) fed blood glucose of Mafa $\mathrm{KO}(n=8)$ and wild-type $(n=11)$ mice at $(\mathbf{m}, \mathbf{n}) 7$ and 12 and (o) 14 weeks of age. (p) $\mathrm{HbA}_{1 \mathrm{c}}$ of Mafa $\mathrm{KO}(n=5)$ and wild-type $(n=4)$ mice at

decreased in Mafa KO islets (Fig. 3b). Interestingly Slc16a1, which is ubiquitously expressed in most tissues but specifically repressed in adult beta cells (a 'disallowed' gene) [34, 35], was upregulated in Mafa KO islets (Fig. 3a). In parallel, the expression levels of Mafb, Pax4, Ngn3 and Sox9, factors
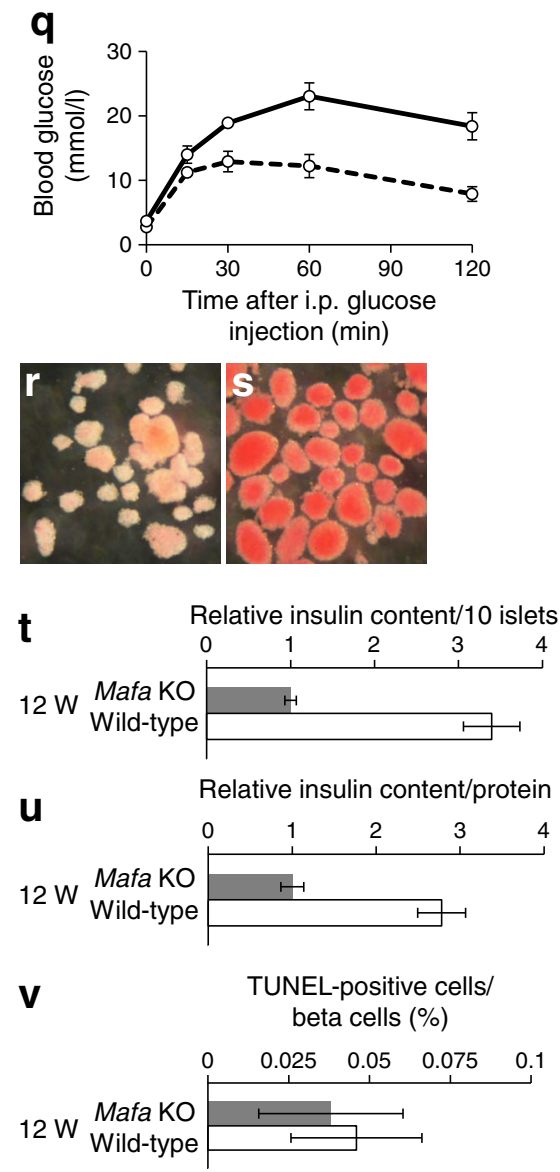

18 weeks of age. To convert values for $\mathrm{HbA}_{1 \mathrm{c}}$ in $\%$ into $\mathrm{mmol} / \mathrm{mol}$, subtract 2.15 and multiply by 10.929 . (q) The results of i.p. glucose tolerance testing of Mafa KO (solid line) and wild-type (dashed line) mice at 10 weeks of age ( $n=4$ for both of genotypes). ( $\mathbf{r}-\mathbf{u})$ Analysis of the islets isolated from Mafa $\mathrm{KO}$ and wild-type mice at 12 weeks of age. $(\mathbf{r}, \mathbf{s})$ Dithizone staining of the Mafa KO (r: $n=3)$ and wild-type (s: $n=3)$ islets. (t) Relative insulin content of 10 islets and (u) relative insulin content per protein ( $n=10$ from two mice). (v) Proportion of TUNELpositive cells per beta cell in the Mafa KO ( $n=9,915$ from seven mice) and wild-type ( $n=11,767$ cells from 10 mice) mice. The means \pm SEM are shown. Scale bars, $20 \mu \mathrm{m} .{ }^{* *} p<0.01,{ }^{*} p<0.05$

transiently expressed in endocrine progenitors of the embryonic pancreas [21, 22], were increased in the Mafa KO islets (Fig. 3b). In addition to these changes in islet factors, relative increases in the expression of Sox2, Nanog and $\mathrm{Mycl}$ were detected, although with the exception of $\mathrm{Mycl}$, these were 
Fig. 2 Dedifferentiation of beta cells in the Mafa KO islets. (a-d) Mafa $\mathrm{KO}$ and wild-type islets stained for insulin (INS; red) and SYP (green, a: $n=9 ; \mathbf{b}: n=12$ ) or CHGA (green, $\mathbf{c}: n=9 ; \mathbf{d}: n=12$ ) at 12 weeks of age. Scale bars, $20 \mu \mathrm{m}$. (e, f) TEM of islets isolated from (e) five Mafa $\mathrm{KO}$ and (f) three wild-type mice at 7 weeks of age ( $n=3$; scale bars, $1 \mu \mathrm{m}) .(\mathbf{g}-\mathbf{n})$ Lineage tracing study. (g) RIPCreER mice were crossed with Rosa-YFP mice in the background of Mafa $\mathrm{KO}$ or control wild-type mice to generate MafaKO;RIPCreER;Rosa-YFP and wild-type;RIPCreER;RosaYFP mice. (h-k) These mice were administered tamoxifen at 4 weeks of age, and pancreas sections were analysed by immunohistochemistry at 12 weeks of age. Red: INS (h: $n=$ $8 ; \mathbf{i}: n=12)$ or glucagon (GCG) (j: $n=8 ; \mathbf{k}: n=12)$; green: YFP (hk). Higher magnification of the demarcated areas to the right shows beta cells with reduced/lost expression of insulin (h) that were converted to glucagon-expressing cells ( $\mathbf{j}$; arrows) over 8 weeks in Mafa KO mice. (l) Body weight, (m) fed blood glucose and (n) fasting blood glucose of the MafaKO;RIPCreER;Rosa-YFP (grey bars; $n=13$ ) and wildtype;RIPCreER;Rosa-YFP (white bars; $n=16)$ mice. Scale bars, $20 \mu \mathrm{m}(\mathbf{h}-\mathbf{k})$ and $10 \mu \mathrm{m}$ (higher magnification)

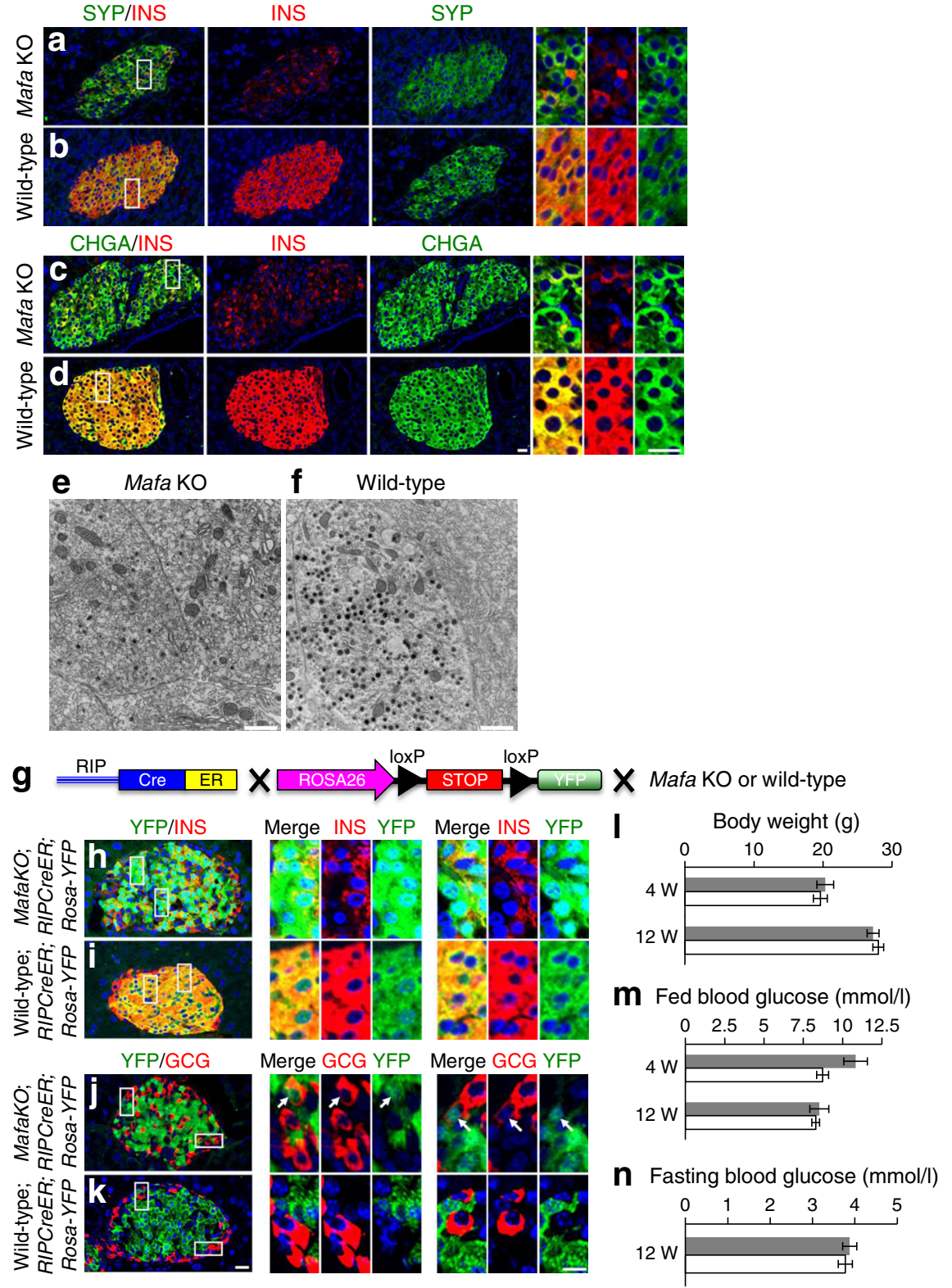

several orders of magnitude below the functional levels seen in embryonic stem cells (Fig. 3c, ESM Fig. 3i). These results suggest that beta cell dedifferentiation induced the upregulation of molecules, including in the 'disallowed genes in mature beta cells' [34, 35], and reduced the expression of key molecules for beta cells, leading to altered metabolism and impaired insulin release in Mafa $\mathrm{KO}$ beta cells.

The expression of DNA methyltransferases was analysed because the loss of DNA methyltransferase (DNMT) 1 or DNMT3a results in a conversion from beta cell to alpha cell [4, 5]. Dnmt1 and Dnmt3a expression was decreased in the Mafa KO islets (Fig. 3d), whereas Dnmt3l was upregulated, which may play a role in the maintenance of hypomethylation at promoters of bivalent developmental genes [36].
Beta cells in $d b / d b$ and MLD-STZ mice are dedifferentiated The beta cells in a diabetes mouse model were subsequently examined because MafA expression was reduced in the beta cells of diabetic mice (ESM Fig. $4 a, b)$ and it was more sensitive to glucotoxic change than PDX1 (ESM Fig. 4c, d) as previously described [25, $26,37]$. In $d b / d b$ mice, the beta to alpha cell ratio was reduced and hormone-negative cells were increased in the islets (ESM Fig. 4e-h). The $d b / d b ; R I P C r e E R ; R o s a-Y F P$ mice and control $\mathrm{db} / \mathrm{m}$;RIPCre;Rosa-YFP mice were generated and injected with tamoxifen at 8 weeks of age (Fig. 4a). A pancreas analysis at 12 weeks of age detected YFP-expressing cells with reduced or no insulin expression, which is similar to the beta cells of MafaKO;RIPCreER;Rosa-YFP mice (Fig. 4b, c). In these 
Fig. 3 Gene expression in Mafa KO islets. The mRNA expression of the indicated molecules in the islets isolated from Mafa $\mathrm{KO}$ mice relative to the wild-types at 7 weeks of age analysed by qRTPCR. (a) Molecules implicated in beta cell function ( $n=4$ for InsI, Ins2, Snap25 and Epac2 (also known as Rapgef4); $n=6$ for Pcsk $1 ; n=3$ for other molecules).

(b) Transcription factors ( $n=6$ for Foxol, Arx and Brn4 (also known as Pou3f4); $n=4$ for Pdxl, Neurod1, Pax6, Mafb, Ngn3, Sox9; $n=3$ for other factors). (c) Expression of the indicated factors in the islets of Mafa $\mathrm{KO}$ islets (grey bars) and mouse embryonic stem cells (mES; white bars) relative to the wild-type islets $(n=3)$ ( $\log _{10}$ transformed). Oct4 is also known as Pou5f1. (d) Expression of Dnmts $(n=5$ for Dnmt1 and $D n m t 3 a ; n=4$ for Dnmt3b and Dnmt $3 l$ ). The means \pm SEM are shown. ${ }^{*} p<0.05,{ }^{* *} p<0.01$ relative to wild-types a

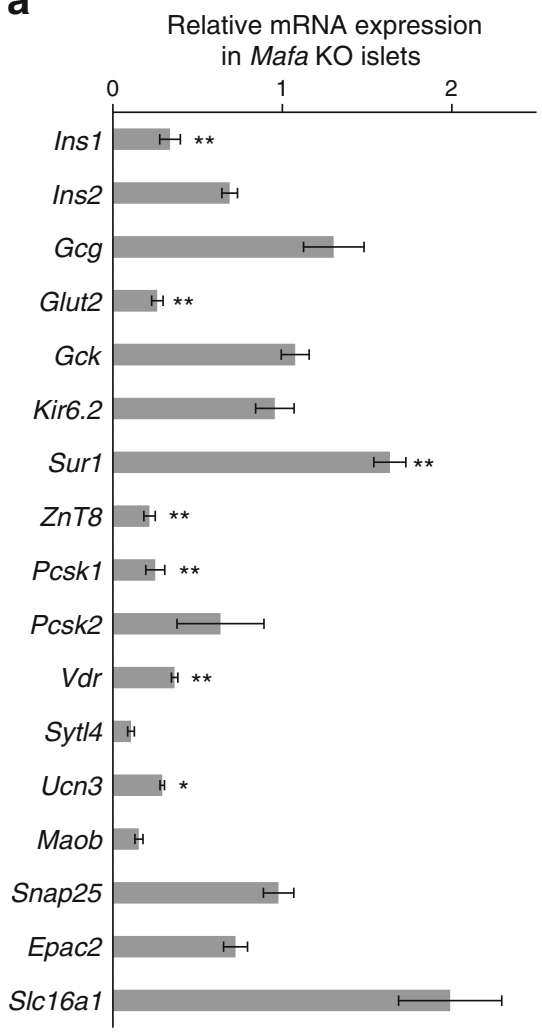

C

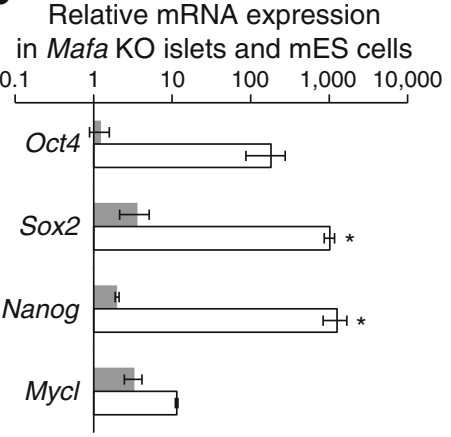

b

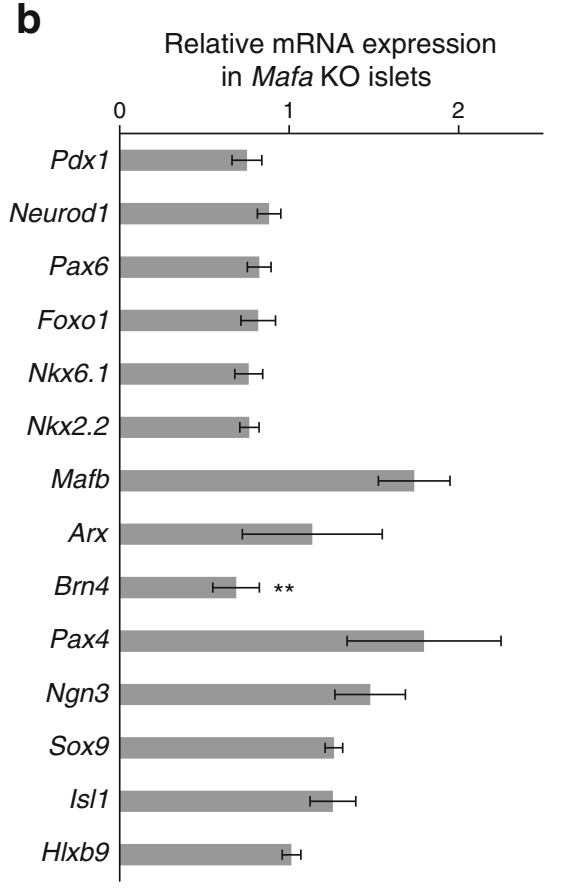

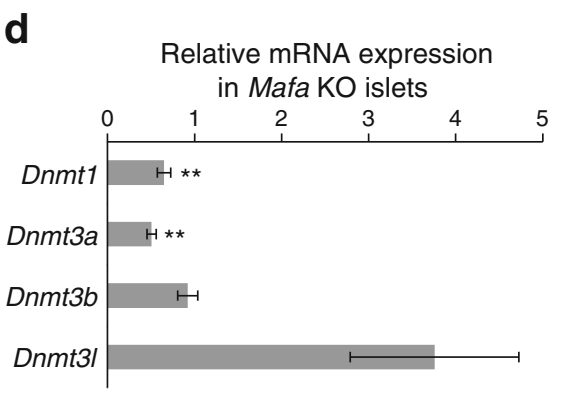

pancreases, glucagon ${ }^{+} \mathrm{YFP}^{+}$cells were observed in $2.1 \%$ of the total glucagon ${ }^{+}$cells $(n=1,165)$ compared with $0.6 \%$ in controls $(n=486)$, suggesting that beta cell conversion to glucagon ${ }^{+}$occurs in the islets of $d b / d b$ mice (Fig. $4 \mathrm{~d}-\mathrm{h}$ ). These insulin ${ }^{-}$beta cells and glucagon ${ }^{+}$beta cells were also observed in diabetic wildtype;RIPCreER;Rosa-YFP mice 3 weeks after injection of MLD-STZ (ESM Fig. 5). In this diabetes mouse model, glucagon ${ }^{+} \mathrm{YFP}^{+}$cells were observed in $2.8 \%$ of the total glucagon $^{+}$cells $(n=696)$ and these cells were $0.2 \%$ of the total glucagon ${ }^{+}$cells in the controls $(n=424)$. Similar results were observed in MafaKO;RIPCreER;Rosa-YFP mice with or without MLD-STZ injections, suggesting the importance of MafA loss in this phenomenon (ESM Fig. 6).
To analyse the dedifferentiation of beta cells in the diabetes mouse model, Mafb expression in the islets was examined using Mafb-GFP reporter mice in which the coding region of $M a f b$ is replaced by GFP such that GFP expression is driven by the endogenous $M a f b$ promoter (Fig. 5a) [28]. In the controls, Mafb-GFP was localised exclusively in the alpha cells (Fig. 5c, e, k, m). Mafb-GFP mice administered MLD-STZ had elevated fasting glucose levels 3 weeks after injection (Fig. $5 \mathrm{f}-\mathrm{i}$ ), and the pancreatic tissue clearly demonstrated a reduced beta to alpha cell ratio and increased GFP expression in non-alpha cells (Fig. 5b-e). GFP expression was observed in $36.1 \%$ of beta cells in MLD-STZ $(n=1,016)$ compared with $0.5 \%$ of beta cells in the controls $(n=1,907)$. Diabetic $d b / d b$;Mafb-GFP mice also 


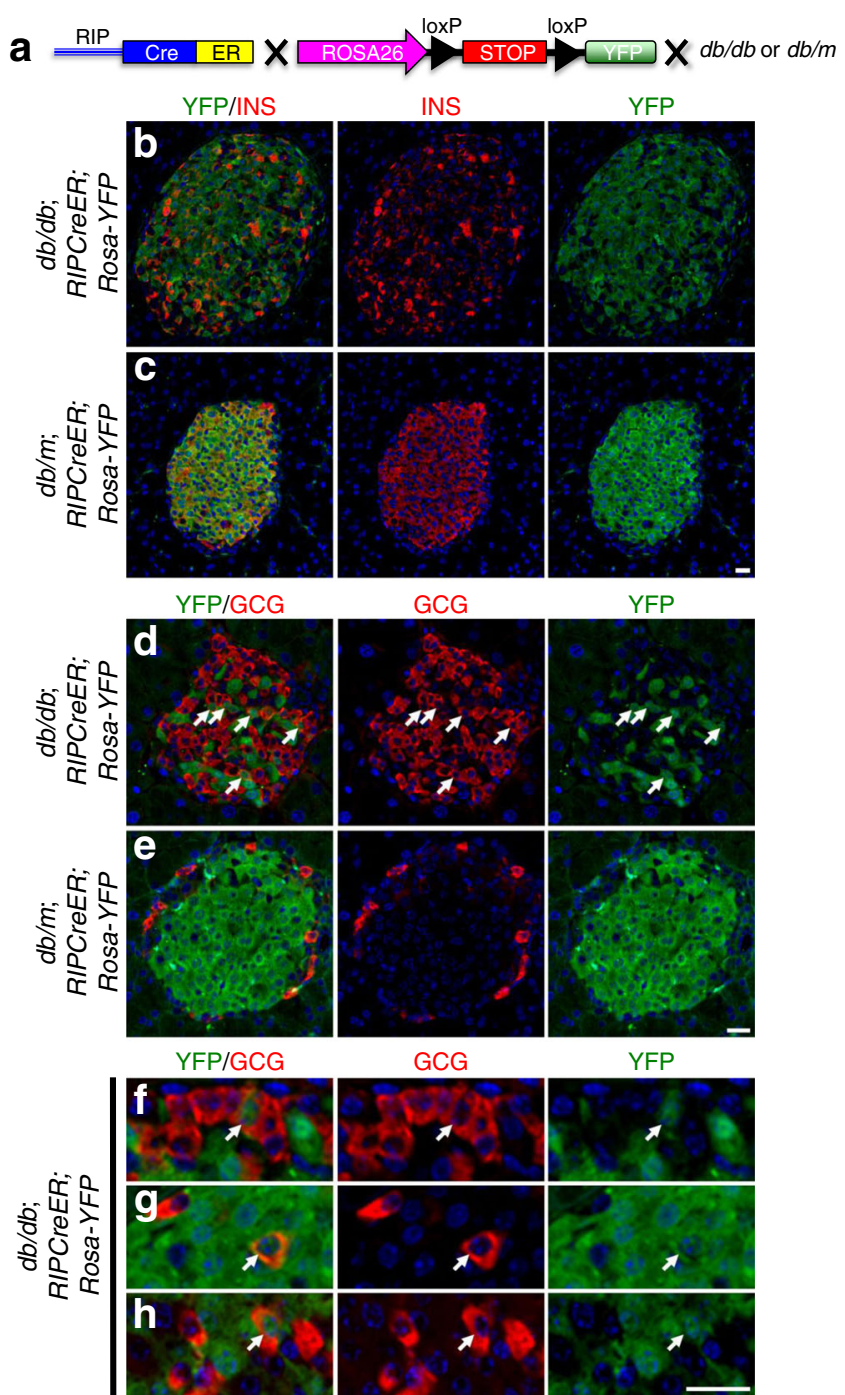

Fig. 4 Beta cell fate in the $d b / d b$ islets. (a) RIPCreER mice were crossed with Rosa-YFP mice in the background of $d b / d b$ or control $d b / m$ mice to generate $\mathrm{db} / \mathrm{db}$;RIPCreER;Rosa-YFP and control $\mathrm{db} / \mathrm{m}$;RIPCreER;Rosa$Y F P$ mice. (b-e) These mice were administered tamoxifen at 8 weeks of age, and the pancreas sections were analysed by immunohistochemistry at 12 weeks of age. Red: insulin (INS; b: $n=5 ; \mathbf{c}: n=3$ ) or glucagon (GCG; d: $n=5 ; \mathbf{e}: n=3$ ). Green: YFP. (f-h) Higher magnification of the $d b / d b ; R I P C r e E R ; R o s a-Y F P$ islets stained for GCG (red) and YFP (green). (d,f,g,h) Arrows indicate beta cells converted to glucagon-expressing cells. The means \pm SEM are shown. Scale bars, $20 \mu \mathrm{m}$

demonstrated glucagon ${ }^{-} \mathrm{GFP}^{+}$cells (Fig. 5j-m). GFP expression was observed in $39.6 \%$ of beta cells in $d b /$ $d b$ mice $(n=1,522)$ compared with $1.5 \%$ in controls $(n=$ 1,158). These results suggest that the Mafb promoter was activated in compromised beta cells in vivo. The expression of $M a f b-G F P$ in the beta cells was not observed in the Mafb-GFP mice fed a HFD from 424 weeks of age (data not shown), indicating that the increased Mafb promoter activity in beta cells was involved in dedifferentiation.

\section{Discussion}

In this study, we investigated the phenotypic plasticity of beta cells and the regulatory role of the key beta cell transcription factor MafA in this process. Our results provide strong evidence that MafA is critical for the maintenance of the mature beta cell phenotype. MafA loss primarily resulted in dedifferentiation of beta cells and upregulation of "beta cell disallowed genes'. These findings are directly relevant to human type 2 diabetes because MafA downregulation occurs in most of the compromised beta cells $[25,26]$ resulting from reactive oxygen species through increased c-Jun expression [38].

This study clearly and mechanistically demonstrated the dedifferentiation of beta cells in Mafa KO mice, $d b / d b$ diabetic mice and mice treated with MLD of STZ through lineage tracing experiments. Dedifferentiation of beta cells is a topic of current islet research, and important contributions have been published by several groups in recent years during the course of this study $[8,13,14]$. In addition, Hang et al showed that MafA is required in adult beta cells for the maintenance of their phenotype but not for their initial differentiation [31], which is consistent with our results.

Recent systemic genome-wide analyses identified a subset of genes that are repressed specifically in beta cells [34, 35]. Inactivation of these genes ensures that beta cells efficiently and adequately secrete insulin in response to glucose stimulation. Their repression is differentiation-dependent and established during postnatal maturation, and immature beta cells express these 'disallowed genes' [34, 35]. Slc16a1 is one of the most disallowed genes in beta cells, and it was upregulated in dedifferentiated 'empty' beta cells of Mafa KO mice. This result is supported by the increased expression of factors such as MafB that are transiently expressed in endocrine progenitors of the embryonic pancreas. MafB is also upregulated in the dedifferentiated beta cells of beta cellspecific Foxol $\mathrm{KO}$ mice with metabolic stress and $P d x \mathrm{l}$ KO mice [8, 14], and induces genome-wide changes in DNA methylation [39]. Thus, MafB may be responsible for the upregulation of molecules including those beta cell disallowed genes in dedifferentiated beta cells by inducing epigenetic changes, which have been shown to regulate Slc16a1 expression [35, 40].

Reversing the dedifferentiation of insulin-negative beta cells restores beta cell function after the normalisation of hyperglycaemia [13, 33], suggesting that beta cells are plastic until a certain stage. Our study suggests that inducing the redifferentiation of beta cells using extrinsic factors may be an effective treatment for diabetes and MafA may be a potential therapeutic target.

The mechanism for the formation of abnormal islet architecture in diabetes was not fully elucidated by this study. As a limited number of former beta cells were converted into alpha cells and alpha cell proliferation was not accelerated in Mafa 
Fig. $5 \mathrm{MafB}^{+}$glucagon ${ }^{-}$cells and $\mathrm{MafB}^{+}$insulin ${ }^{+}$cells in the islets of MLD-STZ and $d b / d b$ mice. (a) $M a f b-G F P$ reporter mice were used, in which GFP expression is driven by the endogenous $M a f b$ promoter $(\mathbf{b}-\mathbf{e})$. The expression of MafB-GFP in the alpha (b: $n=6 ; \mathbf{c}: n=5)$ or beta (d: $n=6 ; \mathbf{e}: n=5)$ cells of (b, d) MLD-STZ and (c, e) control mice. (f) Body weight, (g) fasting blood glucose and (h) fed blood glucose of the $M a f b-G F P$ reporter mice with (grey bars) or without (white bars; control) MLD-STZ before the injection (Day 0) and 3 weeks after the injection (Day 19). (i) Results of the i.p. glucose tolerance testing 3 weeks after the injection (solid line: $n=9$ for MLD-STZ; dashed line: $n=7$ for control mice). (j-m) MafB-GFP expression in the alpha $(\mathbf{j}: n=3 ; \mathbf{k}$ : $n=3)$ and beta (l: $n=3 ; \mathbf{m}: n=3)$ cells of (j, l) $d b / d b$ and (k, m) control ( $d b / m$ or wild-type) mice. (n) Body weight, (o) fasting blood glucose and (p) fed blood glucose of the $d b / d b ; M a f b-G F P(n=6)$ and control $(n=5)$ mice. In both types of diabetes mouse model, MafB-GFP was observed in the glucagon ${ }^{-}$and insulin ${ }^{+}$cells in the islets. Green: GFP; red: glucagon $(\mathrm{GCG}[\mathbf{b}, \mathbf{c}, \mathbf{j}, \mathbf{k}])$ and insulin (INS $[\mathbf{d}, \mathbf{e}, \mathbf{l}, \mathbf{m}])$. The means \pm SEM are shown. Scale bars, $20 \mu \mathrm{m}$ a
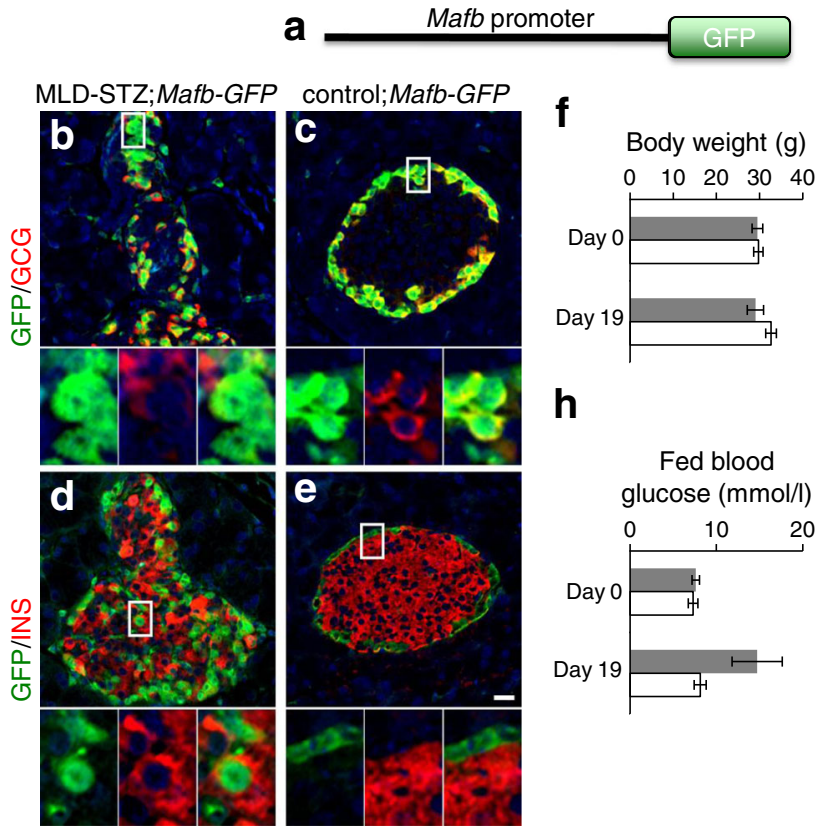

$9 \quad$ Fasting blood g glucose $(\mathrm{mmol} / \mathrm{l})$

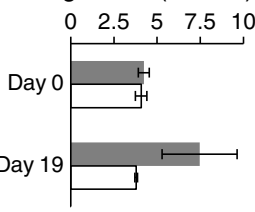

h
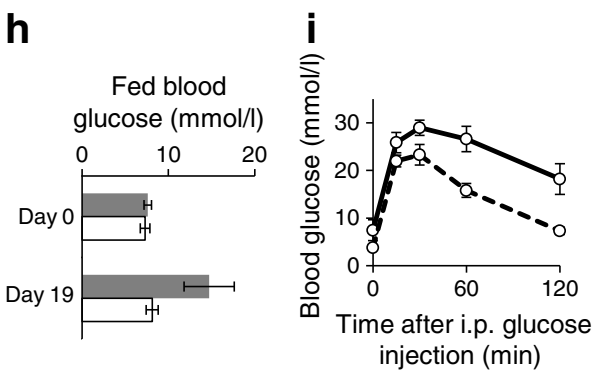

$d b / d b ; M a f b-G F P$

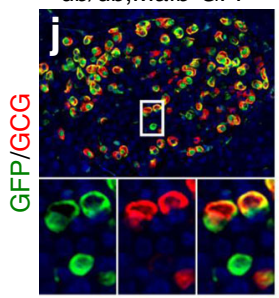

control;Mafb-GFP

\section{n}

Body weight $(\mathrm{g})$
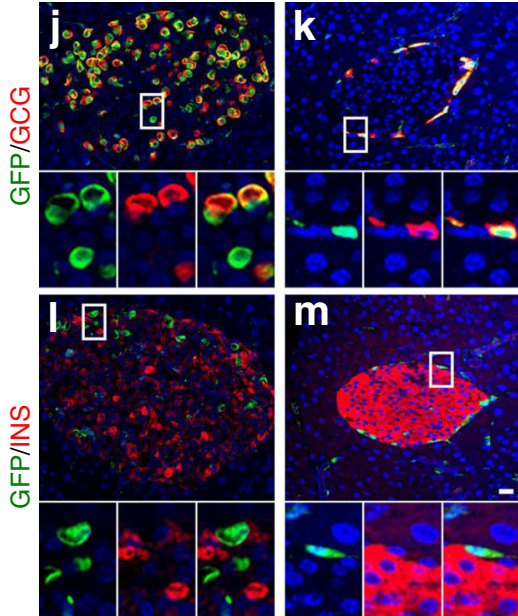

$m$

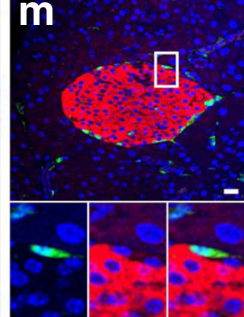

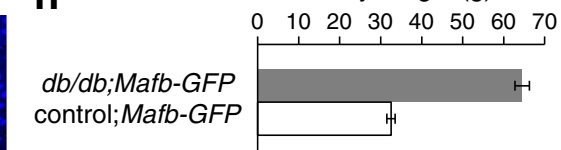

o

Fasting blood glucose (mmol/l)

$\begin{array}{llllll}0 & 2.5 & 5 & 7.5 & 10 & 12.5\end{array}$

$d b / d b ; M a f b-G F P$ control;Mafb-GFP

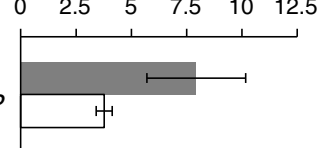

p

Fed blood glucose (mmol/l)

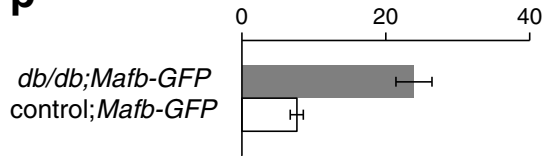

$\mathrm{KO}$ islets, a tentative interpretation for the reduced beta to alpha cell ratio is a reduction in the proliferation of beta cells $[31,32]$, or an as yet unknown mechanism. The lineage tracing of other cell types may provide novel aspects. Additional specifically repressed genes in beta cells, including Ldha, c-Maf (also known as Maf) and hexokinase 1, have been reported [34, 35]. An analysis of these 'disallowed genes' in the pure population of beta cells of mouse models of diabetes with or without beta cell-specific overexpression of MafA may indicate whether or not MafA is directly involved in the repression of these genes and the prevention of beta cell dedifferentiation.

Acknowledgements We thank H. Okochi and T. S. Hamazaki (National Center for Global Health and Medicine, Tokyo, Japan) for the mouse embryonic stem cells, Y. Noda (Jichi Medical University, Tochigi, Japan) for revising the manuscript and M. Tamura-Nakano (National Center for Global Health and Medicine, Tokyo, Japan) for tissue processing and
TEM. We also thank M. Kawaguchi, H. Udagawa, N. Funahashi, M. Hiramoto and T. Nammo (National Center for Global Health and Medicine, Tokyo, Japan) for useful discussions and D. Suzuki, K. Nagase and N. Ishibashi (National Center for Global Health and Medicine, Tokyo, Japan) for their technical assistance. The manuscript was edited by American Journal Experts (Durham, NC, USA).

Funding This study was supported by JSPS KAKENHI, a grant from the National Center for Global Health and Medicine, the Takeda Science Foundation and Japan Diabetes Foundation (WN). KY was supported by JSPS KAKENHI and a grant from the National Center for Global Health and Medicine.

Duality of interest The authors declare there is no duality of interest associated with this manuscript.

Contribution statement WN contributed to the conception and design of the study, the acquisition, analysis and interpretation of the data and drafting the article. ST contributed to the acquisition of data and revising it critically for important intellectual content. KY contributed to the study design, the data acquisition and construction, and critical revision of the 
manuscript. All authors have approved the final version and $\mathrm{WN}$ is responsible for the integrity of the work as a whole.

\section{References}

1. Takahashi K, Yamanaka S (2006) Induction of pluripotent stem cells from mouse embryonic and adult fibroblast cultures by defined factors. Cell 126:663-676

2. Jopling C, Boue S, Izpisua Belmonte JC (2011) Dedifferentiation, transdifferentiation and reprogramming: three routes to regeneration. Nat Rev Mol Cell Biol 12:79-89

3. Thorel F, Népote V, Avril I et al (2010) Conversion of adult pancreatic alpha-cells to beta-cells after extreme beta-cell loss. Nature 464 : 1149-1154

4. Dhawan S, Georgia S, Tschen SI, Fan G, Bhushan A (2011) Pancreatic beta cell identity is maintained by DNA methylationmediated repression of Arx. Dev Cell 20:419-429

5. Papizan JB, Singer RA, Tschen SI et al (2011) Nkx2.2 repressor complex regulates islet $\beta$-cell specification and prevents $\beta$-to- $\alpha$-cell reprogramming. Genes Dev 25:2291-2305

6. Elghazi L, Weiss AJ, Barker DJ et al (2009) Regulation of pancreas plasticity and malignant transformation by Akt signaling. Gastroenterology 136:1091-1103

7. Landsman L, Parent A, Hebrok M (2011) Elevated hedgehog/Gli signaling causes beta-cell dedifferentiation in mice. Proc Natl Acad Sci U S A 108:17010-17015

8. Talchai C, Xuan S, Lin HV, Sussel L, Accili D (2012) Pancreatic beta cell dedifferentiation as a mechanism of diabetic beta cell failure. Cell 150:1223-1234

9. Collombat P, Xu X, Ravassard P et al (2009) The ectopic expression of Pax 4 in the mouse pancreas converts progenitor cells into alpha and subsequently beta cells. Cell 138:449-462

10. Collombat P, Hecksher-Sørensen J, Krull J et al (2007) Embryonic endocrine pancreas and mature beta cells acquire alpha and PP cell phenotypes upon Arx misexpression. J Clin Invest 117:961-970

11. Zhou Q, Brown J, Kanarek A, Rajagopal J, Melton DA (2008) In vivo reprogramming of adult pancreatic exocrine cells to beta-cells. Nature 455:627-632

12. Jonas JC, Sharma A, Hasenkamp W et al (1999) Chronic hyperglycemia triggers loss of pancreatic beta cell differentiation in an animal model of diabetes. J Biol Chem 274:14112-14121

13. Wang Z, York NW, Nichols CG, Remedi MS (2014) Pancreatic $\beta$ cell dedifferentiation in diabetes and redifferentiation following insulin therapy. Cell Metab 19:872-882

14. Gao T, McKenna B, Li C et al (2014) Pdx1 maintains $\beta$ cell identity and function by repressing an $\alpha$ cell program. Cell Metab 19:259-271

15. Taylor BL, Liu FF, Sander M (2013) Nkx6.1 is essential for maintaining the functional state of pancreatic beta cells. Cell Rep 4:1262-1275

16. Puri S, Akiyama H, Hebrok M (2013) VHL-mediated disruption of Sox 9 activity compromises $\beta$-cell identity and results in diabetes mellitus. Genes Dev 27:2563-2575

17. Tata PR, Mou H, Pardo-Saganta A et al (2013) Dedifferentiation of committed epithelial cells into stem cells in vivo. Nature 503:218-223

18. Nutt SL, Heavey B, Rolink AG, Busslinger M (1999) Commitment to the B-lymphoid lineage depends on the transcription factor Pax5. Nature 401:556-562

19. Gurdon JB, Elsdale TR, Fischberg M (1958) Sexually mature individuals of Xenopus laevis from the transplantation of single somatic nuclei. Nature 182:64-65
20. Olbrot M, Rud J, Moss LG, Sharma A (2002) Identification of betacell-specific insulin gene transcription factor RIPE3b1 as mammalian MafA. Proc Natl Acad Sci U S A 99:6737-6742

21. Nishimura W, Kondo T, Salameh T et al (2006) A switch from MafB to MafA expression accompanies differentiation to pancreatic betacells. Dev Biol 293:526-539

22. Artner I, Hang Y, Mazur M et al (2010) MafA and MafB regulate genes critical to beta-cells in a unique temporal manner. Diabetes 59: 2530-2539

23. Nishimura W, Bonner-Weir S, Sharma A (2009) Expression of MafA in pancreatic progenitors is detrimental for pancreatic development. Dev Biol 333:108-120

24. Kaneto H, Matsuoka TA, Nakatani Y et al (2005) A crucial role of MafA as a novel therapeutic target for diabetes. J Biol Chem 280: $15047-15052$

25. Butler AE, Robertson RP, Hernandez R, Matveyenko AV, Gurlo T, Butler PC (2012) Beta cell nuclear musculoaponeurotic fibrosarcoma oncogene family A (MafA) is deficient in type 2 diabetes. Diabetologia 55:2985-2988

26. Guo S, Dai C, Guo M et al (2013) Inactivation of specific beta cell transcription factors in type 2 diabetes. J Clin Invest 123:3305-3316

27. Zhang C, Moriguchi T, Kajihara M et al (2005) MafA is a key regulator of glucose-stimulated insulin secretion. Mol Cell Biol 25: 4969-4976

28. Moriguchi T, Hamada M, Morito N et al (2006) MafB is essential for renal development and F4/80 expression in macrophages. Mol Cell Biol 26:5715-5727

29. Nishimura W, Eto K, Miki A et al (2013) Quantitative assessment of Pdx1 promoter activity in vivo using a secreted luciferase reporter system. Endocrinology 154:4388-4395

30. Shirato Y, Tamura M, Yoneda M, Nemoto S (2006) Centrosome destined to decay in starfish oocytes. Development 133:343-350

31. Hang Y, Yamamoto T, Benninger RK et al (2014) The MafA transcription factor becomes essential to islet $\beta$-cells soon after birth. Diabetes 63:1994-2005

32. Eto K, Nishimura W, Oishi $H$ et al (2014) MafA is required for postnatal proliferation of pancreatic $\beta$-cells. PLoS One 9: e104184

33. Laybutt DR, Hawkins YC, Lock J et al (2007) Influence of diabetes on the loss of beta cell differentiation after islet transplantation in rats. Diabetologia 50:2117-2125

34. Pullen TJ, Rutter GA (2013) When less is more: the forbidden fruits of gene repression in the adult $\beta$-cell. Diabetes Obes Metab 15:503512

35. Thorrez L, Laudadio I, van Deun K et al (2011) Tissue-specific disallowance of housekeeping genes: the other face of cell differentiation. Genome Res 21:95-105

36. Neri F, Krepelova A, Incarnato D et al (2013) Dnmt3L antagonizes DNA methylation at bivalent promoters and favors DNA methylation at gene bodies in ESCs. Cell 26:121-134

37. Harmon JS, Stein R, Robertson RP (2005) Oxidative stress-mediated, post-translational loss of MafA protein as a contributing mechanism to loss of insulin gene expression in glucotoxic beta cells. J Biol Chem 280:11107-11113

38. Matsuoka TA, Kaneto H, Miyatsuka T et al (2010) Regulation of MafA expression in pancreatic beta-cells in $\mathrm{db} / \mathrm{db}$ mice with diabetes. Diabetes 59:1709-1720

39. Vicente-Dueñas C, Romero-Camarero I, González-Herrero I et al (2012) A novel molecular mechanism involved in multiple myeloma development revealed by targeting MafB to haematopoietic progenitors. EMBO J 31:3704-3717

40. Bramswig NC, Everett LJ, Schug J et al (2013) Epigenomic plasticity enables human pancreatic alpha to beta cell reprogramming. J Clin Invest 123:1275-1284 